\title{
Supporting Nurses' Information Flow by Integrating Paper and Digital Charting
}

\author{
Charlotte Tang and Sheelagh Carpendale \\ University of Calgary, Canada \\ char.tang@ucalgary.ca; sheelagh@ucalgary.ca
}

\begin{abstract}
Information technology has changed the way health care is delivered. Electronic health records which are prevalently deployed to replace or supplement paper documentations have made distributed information access at various points of care and work activity achievable with the use of mobile information devices. Our particular concern is with nurse's information flow, where nurse's notes and observations taken at the point of care feed into the electronic record. In these cases, digital technology has not yet entirely replaced paper and pen, because the latter still provide greater ease and flexibility of use when compared to current digital technologies. Even when mobile digital technology is available, clinicians still prefer creating handwritten notes, and then later manually transposing them into the digital medium. Within this context, we created a prototype that integrated digital paper with electronic health charts to retain the benefits of paper and pen, as well as digital medium. A focus group evaluation of this prototype demonstrated promise and potential for its value in a medical environment.
\end{abstract}

\section{Introduction}

Our work concerns nurse's information flow, where there is discord between the way nurses use pen and paper to record notes and observations taken at the point of care, vs. how they feed this information into the electronic record. The focus on the flow of information from a nurse into the Electronic Health Record (EHR) is a crucial factor in overall nurses' effective collaboration and coordination. If the nurse can enter primary notes and readings during patient contact, the nurse/patient face to face interaction is naturally facilitated (i.e., note taking will not interfere). If the information is entered into the EHR in a correct, complete and timely way, the way other nurses on the current or on a later shift can exploit 
this information (usually asynchronously) is also improved. Of course, the longterm archival record is itself an information source that is used by many others, be it administrators, doctors, and so on. Again correctness, completeness and timeliness is critical.

Currently, many clinicians persistently rely on paper personal artefacts (e.g., personal notes written on a note pad and carried around) that they informally use in the course of their work (Hardey et al. 2000, Fitzpatrick 2004, Tang \& Carpendale 2006). This is despite advances in information technology in the last few decades that have considerably changed the way health care is delivered. The importance of paper personal artefacts to support effective information flow in patient care has been well recognized (Allen 1998, Hardey et al. 2000, Silva et al. 2006). Notably, they offer flexibility of use with ongoing tasks (Luff et al. 1992, Mackay 1999, Sellen \& Harper 2002, Nomura et al. 2006). Newly emerging information can be easily added to these paper artefacts during work in progress. In contrast, the process of updating information via digital devices such as PDAs or Tablet PCs is slower (Silva et al. 2006). Studies also found that nurses relied heavily on their paper personal artefacts when delivering patient care, even when alternative mobile information technology was available, e.g., a computer-onwheels with bedside information access (Tang \& Carpendale 2008). In fact, many nurses in our study ward regarded these paper personal artefacts as indispensible to their nursing work and non-replaceable.

At some point, this information must be fed into the EHRs. Currently, EHRs have increasingly replaced or supplemented paper-based documentation. These digital records are important. They allow quick information access across distributed locations without the need for physical transportation of paper information artefacts, which was often time-consuming, required careful planning, and needed coordination among multiple parties. With the increasing awareness of the importance of having information easily available at points of care for improved patient care, various mobile technological devices have often been introduced, e.g., PDAs, smart phones, Tablet PCs, and computers-on-wheels (Silva et al. 2006, Tang \& Carpendale 2006, Zamarripa et al. 2007). However, these devices rarely afford an intuitive mode of interaction for accessing information at points of care (Cohen \& McGee 2004, Lu et al. 2005). Thus, paper personal artefacts are still heavily in use. Nurses often create these at the beginning of a shift. They extract information from distributed information sources creating a copy on paper. For instance, information source could be computer terminals, individual patient charts, or verbal communication from colleagues (Tang \& Carpendale 2006, 2008).

The mandatory process where nurses document medical information that emerges during nurse's shift in formal records is called charting. Currently, charting is time-consuming and constitutes a considerable segment (around 13$28 \%$ ) of nursing activities (Allen 1998, Strople \& Ottani 2006). As part of their 
information flow, nurses have to manually transpose the information from their handwritten notes on paper artefact into the digital EHR. This is not only timeconsuming but is also prone to errors. (Zamarripa et al. 2007).

Given this context, we set ourselves the research goal to design technological solution to support nurses' actual work practices (Tang \& Carpendale 2006, Vincente 2004) around their information flow. Our prototype allows nurses to maintain their familiar and efficient practice of personalized information recording on a paper-like interface, while integrating their paper inscriptions and direct input into the digital hospital information system. We believe such paper and digital integrated charting has the potential to improve not only charting efficiency, but will increase information timeliness by making that information immediately available to other clinicians. We conducted a focus group study around our prototype as a preliminary step to evaluate its effectiveness in facilitating nurses' information flow practices.

Our technology design is based on the findings from in-depth field studies in a local hospital ward (Tang \& Carpendale 2006, 2008) combined with iterative design discussions with the nurses. To set the scene, we introduce our design process by first briefly recounting the findings of these two studies, paying particular attention to shift changes during which the paper personal artefact is created. These findings are gathered to formulate goals for designing applicable technologies, leading to a description of our integrated charting concept. We then describe the focus group study we conducted to evaluate our technology prototype. This is followed by our findings. Finally, we summarize the lessons learned as adjusted and refined design guidelines for building technologies to support the nurses' information flow practices.

The contributions of this research are:

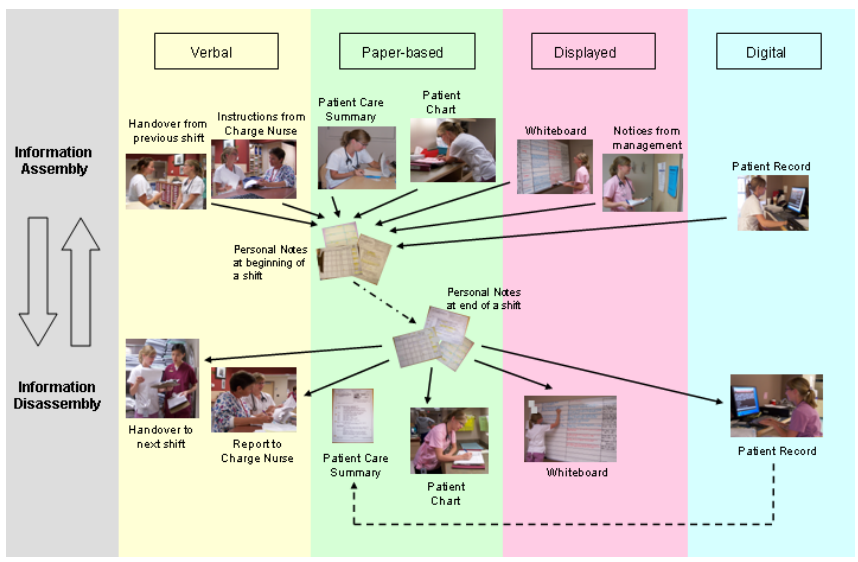

Fig. 1: Incoming nurses acquired information form distributed multimedia information sources (top row), placed the extracted information in their paper personal notes (centre), added new information to personal notes, and finally charted the new information (bottom row). 
- The identification of the need to explore and improve the interrelationships between nurses' informal information practices and the organizational information system in a hospital setting.

- The design and prototyping of a paper and digital integrated charting concept, designed to support informal use of paper personal artefacts as an integral part of the official documentation process.

- The study and analysis of focus group feedback from nurses to evaluate the effectiveness of this integrated charting approach.

- The presentation of a detailed set of design guidelines for future technology development.

\section{Design Process}

In this section we describe how our field studies (Tang \& Carpendale 2006, 2008), and design discussions with the nurses working on the study ward led to our design ideas and the creation of paper and digital integrated charting.

Baseline Observational Study. The first study provided us with a thorough understanding of how information flow took place during nurses' shift change and through their shift, as summarized in Fig. 1 (Tang \& Carpendale 2006). Nurses coming on a new shift had to acquire a good understanding of the current operation in order to carry on the patient care appropriately. To facilitate this, nurses used an intermediary artefact, typically a note sheet which we simply refer to as personal notes (Fig. 1, centre), which has been found to play a crucial role in nursing work. They wrote on their personal notes a consistent set of information that they extracted from distributed multimedia information sources such as patient charts and the EHRs. This graphological practice has been repeatedly reported to help build a mental model of their nursing duties for the shift (Kidd 1994, Tang \& Carpendale 2006). Surprisingly, this manual exercise to record information at shift change was not included in the handoff strategies recommended by Patterson et al. (2004). Nurses also customized their personal notes with individualized layout of information that helped them to retrieve information from their written markings (Fig. 2, left). In the course of their shift work, nurses added newly emerging information to their personal notes and used it to constantly adjust their work plan. Finally, the gathered information had to be properly documented to support continual patient care across consecutive shifts (Fig. 1, bottom).

In brief, nurses relied upon these personal notes for specific functions: as their work plan for nursing work, as their immediate information source and opportune notepad during their shift, and as their information basis for reporting and handover for uninterrupted patient care (Tang \& Carpendale 2008). Thus it is important to design technology to preserve these benefits and to support individual nurses' needs for personalized note taking. 

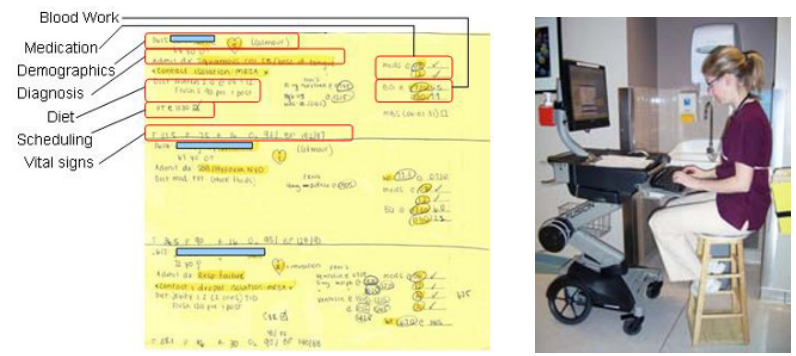

Fig. 2: (Left) Personal notes with customized layout. (Right) A computer-on-wheels.

Studying the Impacts of a Mobile Technology. In the second study we investigated how the deployment of a mobile information technology (a computer-on-wheels as in Fig. 2, right) impacted the nurses' information flow (Tang \& Carpendale 2008). The mobile technology introduced in our study ward was often found not to be used for information access at points of care as intended. Contrasting with the affordances offered by paper notes, these mobile technologies fell short in supporting the important roles necessary for carrying out nursing functions as described above. This motivated us to focus on how the paper and the digital world might be bridged so that information can be converted efficiently across the divide while preserving the benefits of paper use.

\section{Paper and Digital Integrated Charting}

Findings from the field studies were validated by our participants, through proofreading papers and by providing feedback at presentations. Our design ideas evolved iteratively in response to feedback received from the participants through discussions and presentations. To illustrate iterative nature of the design process, the baseline study identified that extracting information from the EHR and writing personal notes on paper was time-consuming and tedious. Thus, we first intended to replace this manual transcription practice with a computer interface which would allow nurses to drag and drop required information from the EHR directly onto a personal notes template and provide features to augment important information such as highlighting or using different colors. These personal notes can be printed, used as immediate information source, and as an opportune notepad adding information as it is acquired during their shift. However, subsequent informal discussion with the nurses indicated that the manual handwriting process at the beginning of a shift was crucial for building a solid mental model of the agenda for their shift (Ash et al. 2004). Similarly, feedback was continuously fed into our design process leading to re-design, re-thinking, or validation of different aspects of our design. Our design goals are: 


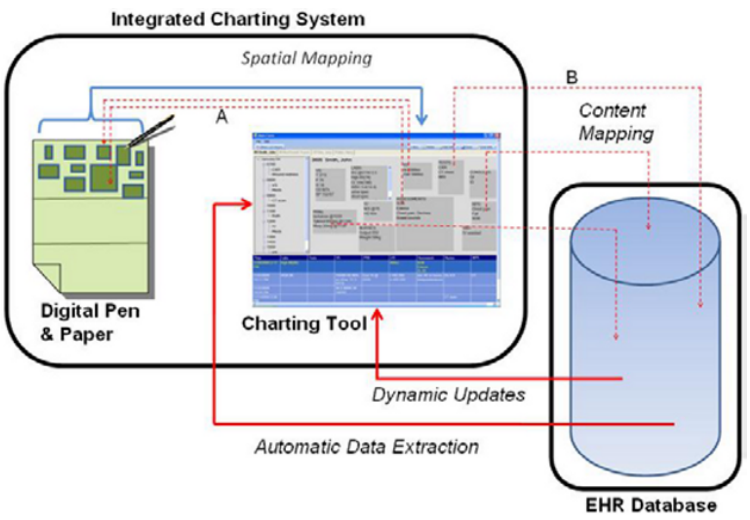

Fig. 3: Design scheme of the integrated charting system.

- To build upon the affordance of paper,

- To support nurses' current information flow practices,

- To use the advantages of EHR to provide instant availability of consistent information to distributed clinicians

\section{Paper and Digital Integrated Charting Overview}

In our paper and digital integrated charting (PDIC) approach, the EHR exists in full functionality, uncompromised and, if desired or if necessitated by circumstance can be used and interacted with as before. In addition, information gathered from the EHR can be organized with digital pen and paper as best suits personal work practices. This digital paper personal information artefact can be used through the shift just as previous personal paper notes were used, recording work done and collecting pertinent information as it arises. A charting interface facilitates the recording of the information generated during the shift into EHR.

A schematic design of the integrated system as integrated with EHR is shown in Fig. 3. It adds two parts to the EHR information system: digital pen and paper technology (Fig. 3, left \& Fig. 4), to allow nurses to use tangible pen and paper as they did previously, and a computer application, Charting Tool (Fig. 3, center \& Fig. 6), for creating digital paper templates and converting handwritten notes into
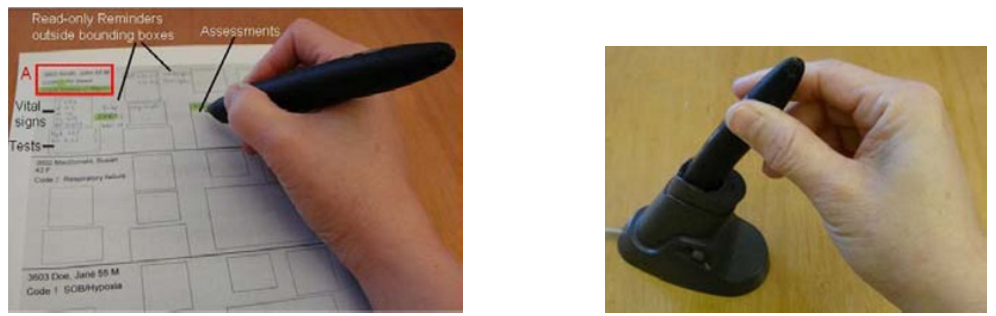

Fig. 4: (Left) Personal notes template printed in digital paper with customized layout of bounding boxes and static information (Right) A digital pen docking station. 
digital text (Fig. 6, top). The dotted lines (A \& B) in Fig. 3 indicate the mappings between these components and are described below.

\section{Paper and Pen Digital Integrated Charting Components}

\section{Digital Paper and Pen}

Our PDIC makes use of the Anoto ${ }^{\mathrm{TM}}$ digital pen and paper technology. Regular paper is printed with Anoto dot patterns which when combined with a pen that contains a camera provides digital functionality. The patterns consist of numerous small black dots that are digitally legible and form an innocuous background that does not interfere with normal use for writing and diagrams. The existence of many patterns can make individual pages uniquely identifiable. The digital pen (Fig. 4, left) contains a digital camera that captures all the markings made on digital paper. The dot pattern on each digital paper indicates the exact position of markings made by a digital pen on the paper. This location-sensitive capability of the digital paper supports creation of distinct regions that can be identified visually for organizing information and computationally when interfacing with the EHR. It is also possible to discriminate between information written in a region or between regions. Thus all notes including information on the exact position the markings on the paper are digitized by and stored in the pen. This information can be downloaded to a computer immediately via Bluetooth technology, or at any point in time using the pen docking station (Fig. 4, right).

Creating personal notes templates. Individual nurses can customize digital paper for their shift by using the charting interface (see Fig. 6) to create and save a template that specifies the information types and their layout. To create a template, an information type, e.g., vital signs, is chosen and a box that bounds the region that will contain this information type appears in the charting interface. It can then be moved and resized as desired. This method provides flexibility for nurses to each has their own type of personal notes and binds the regions on the digital papers with the specified information type. Other information types can be added in the same way until the template layout is satisfactory. Thus the charting interface can be used for creating any number of information layouts. Based on findings that nurses used a layout for all patients and preferred all their patients' information displayed on a single page (Tang \& Carpendale 2006), once a template is created nurses can specify the number of patients and obtain a digital paper that uses their template sized appropriately for their number of patients. For example, Fig. 5 shows a template created on the charting interface (left), and two personal notes for 4 (middle) and 5 (right) patients using the same template for different number of patients. This reflects the way nurses dynamically adjust their personal notes in practice. 

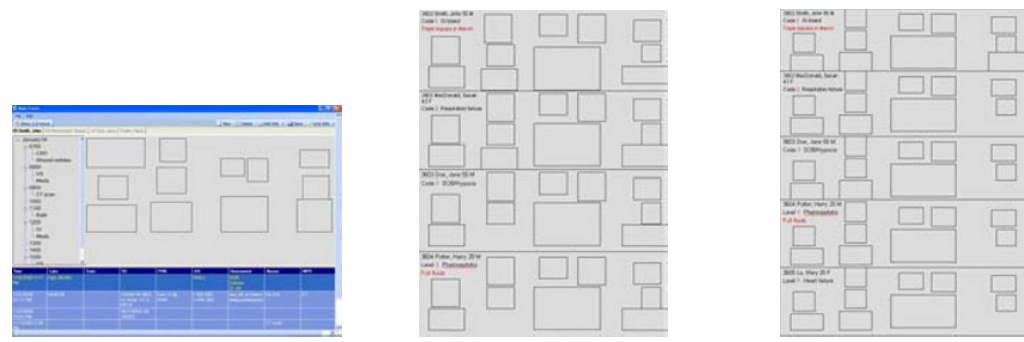

Fig. 5: (Left) Personal notes template created on Charting Interface (Middle \& Right) Personal notes printed for 4 and 5 patients respectively

Using digital-paper personal notes. At the beginning of a shift, nurses print their personal notes based on their template on a digital paper (Fig. 5, middle \& right). Patients' static information such as demographics and medical history can be printed with the template (Fig. 4, left A). Nurses can then use their digital personal notes with their digital pen. If desired they can still highlight important notes with regular highlighters (Fig. 4, left), as has been common practice. Thus these digital personal notes can be used as before to facilitate nurses' work both as a bed-side information look-up, and for recording newly emerging information. For example, as before nurses can record vital signs in the vital signs region.

Nurses' normal practice also includes notes used for references or for personal use that is not intended to be charted. For this, PDIC provides two alternatives: 1) spaces outside the bounding boxes are not charted by default, or 2) bounding boxes can be designated for this specific purpose. Handwritten notes placed in region thus specified will be discarded and will not be converted into digital text for charting, whereas handwriting in the other bounding boxes will be processed and converted into digital characters for charting.

\section{Charting Tool}

The Charting Tool is the software interface between the digital paper and pen and the EHR. It consists of three components (Fig. 6):

- a single interface charting interface for editing and charting (top)

- a quick reference to view up-to-date clinical information (bottom)

- a task timeline to support planning of the shift work (left)

To allow a nurse to focus on one patient at a time, the interface displays information for one specific patient, switching between patients is easy with the tab design (top row).

Charting Interface. The same charting interface is used by nurses to create personalized digital paper notes and to transfer handwritten notes captured with the digital pen to a computer. Connection between the digital pen and EHR is done either via the pen docking station or continually via Bluetooth during nurses' shift. However, to maintain the integrity of the EHR the downloaded information will not be processed until the charting interface is used to verify it. The downloaded notes are converted by handwriting recognition software, into 
digital characters which are displayed in bounding boxes corresponding to the defined regions on the digital paper. In the charting interface these notes can be edited if necessary. Nurses are required to verify and confirm the correctness of the information to be charted before saving into the EHR. Once confirmed, the charting interface bounding boxes are mapped to the corresponding fields in the EHR database (Figure 3, B). Through this charting interface, nurses do not have to navigate through the EHR and instead of manually entering the data for charting they simply verify and edit, potentially saving a lot of nurses' time. The charting interface also allows nurses to chart spontaneous but important information from sources other than their personal notes. This can be added to appropriate bounding boxes or entered into a new clear box. Information entered in this manner also requires verification.

Provision for resizing and relocating the bounding boxes, and for dragging and dropping text between bounding boxes allows nurses to easily move information to the correct bounding box. The interface is also customizable and extensible for other less-frequently used information types in order to meet the needs of the patients. For example, if blood work is not already in the template, nurses can dynamically add a bounding box labelled blood work. Nurses can then type in information pertinent to blood work, and this will be saved to the corresponding field in the EHR along with other information.

Quick Reference. The quick reference provides an at-a-glance overview of the EHR as pertains to the patient in view with most recent information first. This enhances information retrieval from the EHR and can also be used to countercheck if specific data has been charted. New information added to the EHR via the charting interface will be instantly displayed and highlighted in the top row of the quick reference. This quick reference can also be customized in

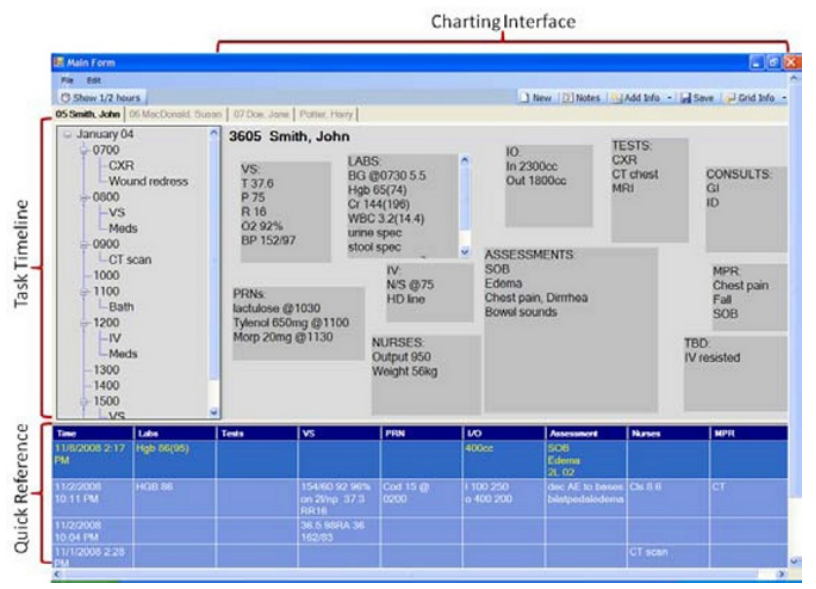

Figure 6: The charting tool consists of a charting interface, a task timeline, and a quick reference to medical records. 
that rows can be highlighted if desired and the columns displayed can be customized for specific types of information.

Task Timeline. On the left hand side of the charting tool is a timeline of tasks to be performed for the currently selected patient that is initially based on timebased information such as medication at certain time intervals that can be automatically extracted from the EHR. This timeline provides an optional planning tool for clinicians to organize their shift work, can be used as a quick overview of shift work, and as a reminder and to-do list. Nurses can also dynamically adjust the timeline (i.e., add, edit, remove, move tasks) in response to changes in their patients' needs and their own temporal horizon in the progress of their shift work (Reddy et al. 2006). In addition, it can also aid reporting to e.g., charge nurse or an incoming colleague, as it displays the tasks that were planned and/or achieved.

\section{The Study}

To evaluate paper and digital integrated charting, we prototyped the basic concepts and ran focus groups with participants from our original study ward. Focus groups were chosen to provide a good initial step in iterating the system design as participants could acquire hands-on experience with the prototype and freely express and discuss their experience and expectations.

\section{Participants}

Focus group participants were nine registered nurses and three nursing students. They all were experienced in preparing and using paper personal notes for their shift work. Because the participants were the targeted end-users of the technology, they were highly motivated to offer their practical experience and expectations of the technology. Six focus groups were conducted in total. Data from one group was not used because participants opted out of key portions of the focus group protocol. The participation in the focus groups was negotiated around existing nurses' schedules. These groups were planned for times when two or more people agreed to participate. The final composition of the focus groups ranged from 1 to 5 participants $(5,3,2,1,1)$ due to unexpected illnesses and family emergencies.

\section{Method and Materials}

Each focus group lasted about an hour and participants engaged in these activities.

1. Creating an individual template. Each participant drew on paper the spatial layout of their own paper personal notes, labelling the respective types of 


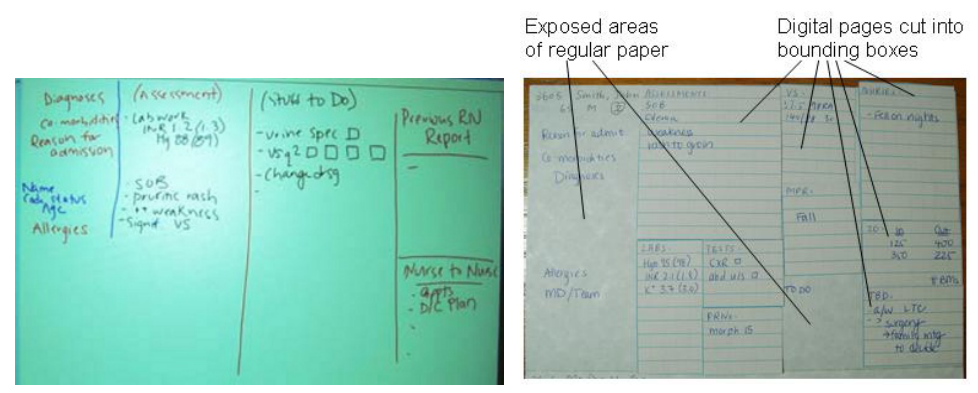

Figure 7: (Left) Collaborative template created on Smart Board. (Right) Corresponding paper prototype created by gluing snippets of (lined) digital paper on a piece of regular paper (plain grey).

information such as medications and assessments. Stationery such as pens, markers and highlighters of different colors were provided. Participants were encouraged to create the template as close as possible to the personal notes they created and used in practice. This activity took less than five minutes.

2. Creating a collaborative template. Participants worked as a group to discuss and design a template for personal notes that was acceptable to all members of the group using either a Smart board or a large flip-chart (Fig 7, left). This group template was needed for use in the next activity. Single participants simply used their own template. This collaborative template can also help inform the design of a generic template for use by nurses who have not yet created their own template, e.g., new nurses.

3. Prototyping personal notes. Pages from Adap ${ }^{\mathrm{TM}}$ Digital Journalx, which were lined and printed with Anoto ${ }^{\mathrm{TM}}$ dot pattern (Fig. 7, right), were used. These pages have the same property that PDIC is based on. Each page is identifiable dues to its dot pattern. Participants then cut pages to create required regions and glued them on a piece of regular paper (Fig. 7, right) to match the layout of the collaborative template from activity 2 (Fig. 7, left). Thus notes and handwriting inside each region created from digital-paper will be captured, digitized and identified for a specific kind of information by a digital pen. Exposed areas of the regular paper (Fig. 7, right) could be used for information not required to be charted.

Minor inconsistencies were found in the information types predefined in the charting interface and those used in the collaborative template created in Activities 2 and 3. For example, separate regions were provided for laboratory work and tests whereas some collaborative templates contained only a combined "labs and tests" information type. Therefore, participants had to slightly adjust the layout of the bounding boxes on the constructed digital paper notes. These constructed digital paper notes were in turn used in the next activity.

4. Exploring the PDIC prototype. The researcher explained how the boxes and areas outside the boxes on constructed digital paper notes interfaced with the charting tool. The charting tool was displayed on a Smart board mounted to the wall (Fig. 8) to offer a clear view to all the participants and to trigger discussions 
incorporating their own practical experience and expectation (Wilson et al. 2006). Participants were frequently encouraged to discuss and comment on their experiences and expectations of the technology to better support their work. How the digital pen and paper work was also explained such as the dot pattern imprinted on

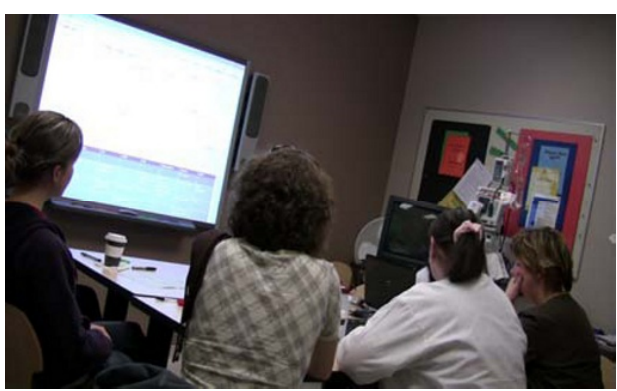

Fig. 8: Participants exploring the integrated charting.

the digital paper, the embedded camera in the digital pen, and why the exact position of markings made on digital paper was known.

Participants then used a digital pen to write on their paper note (Fig. 7, right), pretending that they were preparing and using it as their personal notes in a work shift. They were encouraged to write as they would normally do. Information sheets which contained fabricated medical information, previously compiled with the help of a registered nurse, were provided for participants to prepare the digital personal notes. However, all participants wrote down information from their experience without consulting the information sheets.

The handwritten data was then downloaded via the pen docking station (Fig. 4, right) connected to a computer. The researcher triggered the use of handwriting analysis software to convert the handwritten data into digital text and explained how this happened (i.e. Wizard of $\mathrm{Oz}$ technique) whereas this would be automatically done in a fully-implemented system. Participants could then click the 'Notes' button on the charting tool and see the text converted from their handwriting that was inside the bounding boxes on their digital paper notes. Participants were encouraged to edit and add more detailed information to the displayed text to the point that it was appropriate for charting. They then saved the information after verifying its correctness on the verification screen. Participants were encouraged to explore and comment on all aspects of the charting tool such as the timeline and the quick reference update.

\section{Findings}

The main goal of the study was to identify strengths and weaknesses of the design of paper and digital integrated charting, in particular whether it can facilitate or impede nurses' work practices around their information flow, and to gather suggestions for improving the system design. The study employed a combination of varying-fidelity methods instead of a full-fledged system to demonstrate the design concepts and to actively draw the participants into the design dialog. The 
first three activities, i.e. to draw a personal template, to sketch a collaborative template and to craft a paper prototype of personal notes using digital paper snippets, were conducted to progressively prepare for the last activity which was the focus of the study. Therefore, we briefly highlight the findings from the preparatory activities and concentrate on the findings of the last activity.

\section{Highlights of Preparatory Activities}

Create an individual template. Although the individual templates appeared different, they all contained a consistent set of information: static information (room number, patient name, age, medical history, doctor's name, resuscitation level, allergy, special diet, diagnosis), and dynamic information (vital signs, assessments, I/Os, IV, tests, labs, blood work, medication, previous shift report, and new orders). All our participants confirmed that they created and used paper personal notes for their work.

P1: “... I would never give up my piece of paper. Literally if I lose it, I'd spend like 40 minutes trying to find it. I'll go through every garbage bin 'cause it's like your lifeline. I don't know what to do with myself if I don't have it."

P4: "Because our entire day works on that piece of paper, everything we do, when we need reports, we wouldn't go to the computer. It's all on that [the personal notes]."

P6: "I don't know what to do whenever I lose it [the personal notes]."

Create a Collaborative template. Three focus groups conducted this collaborative activity. Two groups created a new collaborative template based on discussion of the participants. One group selected one of the participants' personal templates as their group template after comparing and contrasting their personal templates and finding that the spatial layout was the only difference among them. Moreover, many participants said that they made changes to their personal templates in terms of both the layout and the information content from time to time when their experience increased and when their patients' needs differed. The activities and discussions around designing a collaborative template indicated that a default but adjustable template could be useful.

Prototyping personal notes. All groups constructed their digital paper notes using snippets of digital paper glued on a regular paper. Three groups conducted this activity based on their collaborative template and the other two were based on the participant's personal template.

\section{Exploring the Paper and Digital Integrated Charting Prototype}

Participants' responses towards our technology design were mostly positive. They were particularly delighted that the design was based on pen and paper interaction. They saw great potential and value of the system in facilitating their work. Several participants asked when the technology would be available for use 
in practice. Yet, they also identified weaknesses of the system and made suggestions for improving the technology design.

\section{Strengths}

Pen and paper. Participants' reliance on their paper personal notes during shift work was in evidence as they referred to personal notes as their 'lifeline' and 'my entire day works on [my personal notes]'. They perceived great benefits in retaining the use of the familiar pen and paper and were excited to see that their handwriting could be converted to digital text without requiring manual re-typing at a computer. They voiced hope that this could improve their charting efficiency as they would only need to edit or add to the converted information.

Single-interface charting. Participants found the ability to chart multiple information types with the same interface useful. They also found the flexibility to dynamically extend the interface to include other kinds of information for charting convenient. They expected that charting would become more efficient and more comprehensive because current tedious and time-consuming navigation of the hierarchical EHR could be minimized. Making current information pertaining to one patient visible on the same interface provided an overview and a more comprehensive picture of patient's condition that could help more easily organize and document different kinds of information gathered during the shift. They also commented about huge time savings to benefit both actual nursing care and hospital finance by paying less overtime work.

'Personal' information space. Some participants wrote everything inside the digital-paper bounding boxes on the constructed digital personal notes whereas others used the space on the regular paper for information that was not meant for charting (Fig. 7, right). The former pointed out the need for a 'personal' information space on their personal notes when they saw the converted text of their personal notes displayed in the charting interface included also their 'personal' information. They explained that this kind of information was vital for accomplishing their nursing work, regardless if it is information extracted from documented sources to serve as reference information, or their own personal opinions towards a patient, e.g., as a reminder of specific ways to deal with the patient, or just some scribbles for catharsis to relieve their stress. In short, all the participants agreed that personal notes should provide 'personal' information spaces for writing down information that will not be charted.

Focus on new information. Participants liked the design that previously charted information is not displayed in the charting interface so that they can always focus on newly emerged information. This is because the charted information would still be readily available in the quick reference table below. One participant suggested including an option for displaying the charted information in the charting interface as long as it was easy to differentiate between the old and new information such as using different color or different font style. 
Quick reference facilitates information retrieval. All the participants regarded the quick reference that offered an at-a-glance view of the archived medical information useful. They found it convenient to look up medical records without having to go through the hierarchical EHR. They also found the dynamic updates in the quick reference table when new information was saved to the EHR helpful in that they could be more aware of up-to-date patient information.

P1: "I like it [quick reference] because... sometimes you don't get a good report from the previous nurse, then you can scroll back to see what the nights [nurse in night shift] think. I wish we had something like this... For example, if a patient fell, the nights tells the days and the days tells the evenings, but when nobody tells anybody anything, you forget that this person may fall. But if there's this thing [quick reference], I can just go back to see what happened and like what's the plan 3 days ago."

P12: "I really like that I don't have to go to different places to look for new things for the patients and it's all here in the blue table [the quick reference table]. I like that!"

Timeline useful to new nurses. Most experienced nurses did not find the task timeline useful for planning and organizing their shift work.

P11 (experienced nurse): "nursing uses flexible process... constantly shifting... no way to plan like this... If you're going to spend time on this, you'll never get to patient care".

However, student nurses liked the timeline. They perceived it a useful tool to help them focus on their work and to allow them visualize their work schedule and work load. Many experienced nurses recalled that they also manually created similar timelines for planning their work when they were new. But they had abandoned this practice as they became more experienced with their nursing duties and were more used to the dynamic and flexible nursing processes (Bjørn $\&$ Balka 2007). Therefore, they believed that the timeline would be a valuable tool for training and educating novice nurses.

\section{Weaknesses and Concerns}

Liability issues. Participants were particularly concerned about their liability for the technology in case they lost their digital pen. Since a digital pen is much more costly than a regular pen which nurses do not worry about losing, participants were worried if they had to be financially responsible for replacement. This led to active discussions on ways to prevent losing the digital pen. Suggestions included carrying it around their neck with a lanyard but they were then worried about the number of gadgets that they had to carry with them. They were also concerned about the technology's costs, durability and environmental issues (e.g., recyclability of digital paper).

Handwriting recognition. Our participants did not express negativity towards the handwriting conversion because their handwriting was generally converted quite well in the study. However as expected, many participants were concerned with the general quality of handwriting recognition. They were also worried about their handwritten symbols and abbreviations (e.g., SOB for short of breath and CXR for chest X-ray) and whether they could be properly transcribed. 
Embedded charting. Participants criticized the system in that it did not facilitate 'flow sheet charting' which is an embedded structure in the EHR. They are required to access this by navigating the information system in order to chart by "point and click" a checklist of information categories in the flow sheet. They suggested having a button that linked with the flow sheet so that they could directly access it without navigating the information system. The researcher proposed using digital-paper flow sheet printed with checkboxes so that charting on flow sheets can be done anywhere and the checked information can be easily transferred to the EHR. This alternative was well received, especially by experienced nurses who prefer paper artefacts to digital medium. But younger nurses found a single-mouse-click link to the digital flow sheet more beneficial.

\section{Suggestions}

Extending the potential of digital pen and paper technology. In view of the potential of digital pen and paper, participants suggested designing the personal notes to include areas for other documentation and reporting purposes in addition to EHR charting. As currently, nurses have to write or verbally report the same information multiple times in different places such as in patient charts, verbal shift reports and large whiteboards, requiring much mobility and redundancy of effort. Therefore, they considered it a great value if they only needed to write once on their personal notes and the information would be saved or displayed (with or without converting to digital text) instantly in different media. For example, participants could write a shift report on their personal notes for relevant clinicians such as their incoming colleague or charge nurse to retrieve at a computer. Participants perceived significant savings in time and mobility when they no longer needed to look for people in order to give or receive reports.

Support both customizable and customized interfaces. Many participants resented that they always had to navigate to their last visited screen every time they logged on the EHR. They were also frustrated that the current EHR did not allow them to keep their customized views of information which would be automatically reverted to the default view when they logged off the system. They estimated that this practice of logging on and off the EHR took place 50 to 100 times per shift. Thus they emphasized it is imperative that our system is designed to allow them to continually use their customized views and to display their last visited screen upon logging on. They also expected that these features would greatly improve their work efficiency.

\section{Discussion}

Integrating the effective aspects of current work practices with the advantages of the EHR is the goal of our Paper and Digital Integrated Charting. Thus this research commenced with observational studies to form a thorough understanding of current work practices. The evidence from our field studies indicated that while 
nurses' use of pen and paper held many advantages, it was in conflict with the hospitals' goal of the consistency of the EHR. This led us to design technology using digital pen and paper so that handwritten notes can be easily digitized, thus working towards the goal of integrating the best of both directions.

The focus group study we conducted using a prototype developed with mixedmethod approach provided encouraging feedback. For example, "This is really quite exciting and I know there're glitches that are not working as well as we'd like. But we're moving towards the right direction" (P11). While weaknesses were identified and concerns were expressed, the feedback gathered indicated potential of PDIC and gathered together provides the following set of refined guidelines.

Support flexibility and personalization. Participants in general were most pleased by the potential for both supporting their current work needs and having the flexibility to support changing work practices.

- The use of flexible templates, as exemplified by the use of bounding boxes to create identifiable and recognizable interface components that can be resized and reorganized to match personal preferences provides both consistency for the computational interface and individuality for personal use.

- Familiarity with interfaces can facilitate work (Kidd 1994). Thus it is important that the customized interfaces can be saved for ongoing and future use.

Facilitate information entry and retrieval. Participants discussed several aspects about current systems that were time-consuming from disseminating information to multiple media to repeated clicking in the EHR. They were hopeful that PDIC would provide considerable time savings.

- Providing navigation that is based on recognizable interface components which have established system mappings has considerable potential for streamlining information updating and retrieval.

- When possible embed required information structures (e.g., flow sheet, multidisciplinary report) within an integrated interface.

Provide an overview. Participants liked being able to see the information gathered from their digital personal notes in conjunction with the timeline and quick reference. They particularly appreciated the quick reference and its instant update on information verification and entry.

- Combining several information representations in a customizable interface avoids problems with fragmented information that has been scattered over different places and has been found to impede the building of mental model (Ash et al. 2004).

- Viewing a comprehensive set of information can provide feedback on what has been done and still needs to be done thus greatly facilitating the collaborative effort across shifts for providing quality patient care, as well as the charting process and its quality. 
- Enriching the information presentation, such as including the timeline which was found to help novice nurses plan, organize and focus on their work, may further enhance the learning outcome and the work efficiency.

Support 'personal' information use. Personal notes, as our participants indicated, are created and used by their owner so are inherently personal and traditionally contain both archival and non-archival information that is only intended for personal use. However, with the use of digital pen and paper, the real 'personal' information would run the risk of being publicized over the digital medium.

- Some information spaces should be assigned strictly for 'personal' use only without leaving any digital trace. Otherwise, the technology would fail to support individual needs and may experience adoption resistance.

Safeguard the accuracy of information. This factor is always a concern for health care systems and our nurse participants are no exceptions. Many discussions and comments focused on this topic primarily in three ways.

- The system must always instigate a mandatory, yet lightweight, verification safeguard before information is saved to the database to uphold the integrity of information accessible by distributed clinicians.

- The system should provide an option of keeping a cache of personal notes information that can be retrieved by its owner. This will be useful in case the frontline information artefact needs to be reproduced.

- The reliability of handwriting recognition is a justifiable concern. However, the use of mandatory verification makes this feasible and advances in handwriting recognition technology, the captured, digitized handwriting can be converted into digital text with increasing accuracy, narrowing the divide between paper and digital medium.

Support system dispersal. Our participants noted, and is confirmed in previous studies, that they both retrieve and disseminate information from/to multiple sources. These rich varieties of information artefacts in medical settings facilitate various groups of clinicians to accomplish many different goals (Bardram \& Bossen 2005). Thus it is not uncommon that the same information has to be inputted in multiple media. This practice undoubtedly cost time and effort.

- An integrated charting system that is linked with other information artefacts and displays could minimize redundancy of effort (Cabitza et al. 2005) and to provide information in multiple modals and representations (Reddy et al. 2001). This in turn will benefit patient care.

\section{Conclusion and Future Work}

Based on previous studies on nurses' information flow, we have designed, prototyped and studied a paper and digital integrated charting solution that offers: 
- manual transposing of information from multimedia sources to a digital paper notes for building a mental model and planning of the shift work,

- the ability for individual nurses to customize their digital personal notes for facilitating information retrieval,

- portable, flexible and low-cost use of digital paper notes to support bedside information access and note-taking,

- easy transfer of information from digital paper notes to the EHR to provide timely, low-cost and continuous information flow,

- the use of personal notes as information basis for reporting in non-digital media such as in verbal shift reports,

- a quick reference to archived medical information, and

- a timeline for visually planning work.

Through focus groups of practising nurses, we studied this technology and obtained valuable feedback on the benefits they perceived and well-articulated suggestions for improving the system. Together, they helped to refine our set of design guidelines that other researchers and designers may find helpful in their specific settings. In order to discover how to best support nurses' work practices, we have taken an iterative design, prototyping and evaluation approach. The next step of this research would be to re-design and re-implement the integrated charting system based on the refined guidelines.

\section{Acknowledgement}

This paper has greatly benefitted from the valuable input of Saul Greenberg. We also thank the nursing staff at W21C, Foothills Hospital, Calgary, and our funding agencies, AIF, iCore, NERSC, and SMART Technologies for their support.

\section{References}

Adapx. http://www.adapx.com, on Jan 20, 2009.

Allen, D. (1998). Record-keeping and routine nursing practice: The view from the wards. Journal of Advanced Nursing 27:1223-30.

Anoto Technology. http://www.anoto.com on Jan 20, 2009.

Ash, J. S., Berg, M. and Coiera, E. (2004). Some Unintended Consequences of Information Technology in Health Care: The Nature of Patient Care Information System-related Errors. Journal of the American Medical Informatics Association 2004; 11 (2): 104-112.

Bardram, J. and Bossen, C. (2005). A Web of Coordinative Artifacts: Collaborative Work at a Hospital Ward. Proc. of GROUP, pp. 168-176.

Bardram, J. and Bossen, C. (2005). Mobility Work: The Spatial Dimension of Collaboration at a Hospital. JCSCW 14(2), pp. 131-160.

Bjørn, P. and E. Balka (2007). Health Care Categories have Politics too: Unpacking the Managerial Agendas of Electronic Triage Systems. Proc. of ECSCW, pp. 371-390.

Cabitza, F., Sarini, M., Simone, C. and Telaro, M. (2005). When Once Is Not Enough: The Role of Redundancy in a Hospital Ward Setting. Proc. of GROUP, pp. 158-167. 
Cohen, P. and McGee, D. (2004). Tangible Multimodal Interfaces for Safety-Critical Applications. Communications of the ACM Jan 2004, Vol. 47 No. 1, pp. 41-46.

Fitzpatrick, G. (2004). Integrated Care and the Working Record. Health Informatics Journal 10(4), pp. 291-302.

Hardey, M., Payne, S. and Coleman, P. (2000). 'Scraps': hidden nursing information and its influence on the delivery of care. Journal of Advanced Nursing, 2000, 32(1), pp.208-214.

Kidd, A. (1994). The Marks are on the Knowledge Worker. Proc. of CHI 1994, pp. 186-191.

Lu, Y., Xiao, Y., Sears, A., Jacko, J. (2005). A Review and a Framework of Handheld Computer Adoption in Healthcare. International Journal of Med Informatics 74(5), pp. 409-422.

Luff, P., Heath, C., and Greatbatch, D. (1992). Tasks-in-Interaction: Paper and Screen Based Documentation in Collaborative Activity. Proc. of CSCW 1992, pp. 163-170.

Mackay, W. (1999). Is paper Safer? The role of Paper flight Strips in Air Traffic Control. ACM Transaction on computer-Human Interaction 1999, 6(4), pp. 311-340.

Nomura, S., Hutchins, E. and Holder, B. (2006). The Uses of Paper in Commercial Airline Flight Operations. Proc. of CSCW, pp. 249-258.

Patterson, E., Roth, E., Woods, D., Chow, R. and Orlando, J. (2004). Handoff strategies in settings with high consequences for failure: lessons for health care operations. International Journal of Quality in Health Care, 16(2), 2004, pp. 1-8.

Reddy, M., Dourish, P. and Pratt, W. (2001). Coordinating Heterogeneous Work: Information and Representation in Medical Care. In Proc. of ECSCW, pp. 239-258.

Reddy, M. Dourish, P. and Pratt, W. (2006). Temporality in Medical Work: Time also Matters. JCSCW 15(1), 2006.

Sellen, A. and Harper, R. (2002). The Myth of the Paperless Office. The MIT Press, Cambridge.

Silva, J., Zamarripa, M., Strayer, P., Favela, J. and Gonzalez, V. (2006). Empirical Evaluation of a Mobile Application for Assisting Physicians in Creating Medical Notes. Proc. of the 12th Americas Conference on Information Systems.

Strople, B. and Ottani, P. (2006). Can Technology Improve Intershift Report? What the Research Reveals. Journal of Professional Nursing, 22(3), 2006, pp. 197-204.

Tang, C. and Carpendale, S. (2007). An Observational Study on Information Flow during Nurses' Shift Change. Proc. of CHI, pp. 219-228.

Tang, C. \& Carpendale, S. (2008). Evaluating the Deployment of a Mobile Technology in a Hospital Ward. Proc. of CSCW, pp. 205-214.

Vincente, K. (2004). The Human Factor - Revolutionizing the Way we Live with Technology. Vintage Canada Edition 2004.

Wilson, S., Galliers, J. and Fone, J. (2006). Not All Sharing is Equal: The Impact of a Large Display on Small Group Collaborative Work. Proc. of CSCW, pp. 25-28.

Zamarripa, M., Gonzalez, V. and Favela, J. (2007). The Augmented Patient Charts: Seamless Integration of Physical and Digital Artifacts for Hospital Work. C. Stephanidis (Ed.): Universal Access in HCI, Part III, HCII 2007, LNCS 4556, pp. 1006-1015. 